\title{
Klaus Busch \\ Mythen über den Weltmarkt - Eine Kritik der theoretischen Grundlagen der Weltsystemtheorie Immanuel Wallersteins
}

\section{Einleitung}

Nach dem Auslaufen ihres Hegemoniezyklus - der obere Wendepunkt ist Anfang der 70er Jahre anzusiedeln - hat die Dependenzheorie am Ende desselben Jahrzehnts in Gestalt der Weltsystemtheorie Immanuel Wallersteins eine neue wissenschaftliche Aufschwungsphase erlebt.

Obwohl die ökonomischen Differenzierungsprozesse in Afrika, Asien und Lateinamerika in den 70er Jahren - Schwellenländer, ölexportierende Entwicklungsländer, medium-income countries (MIC's), low-income countries (LIC's) - den empirischen Erklärungsgehalt der Zentrum-Peripherie-Modelle tief erschïttert haben (vgl. dazu Hurtienne 1981, Barati-Novbari/Seelow 1982), obwohl in zahlreichen Publikationen die Inkonsistenz der Theorie des ungleichen Tausches, die das theoretische Scharnier der weltmarktorientierten Unterentwicklungstheorie bildet, herausgearbeitet worden ist (Lair 1974, Chatelain 1974, Warren 1973, Busch 1973), obwohl es mittlerweile mehrere Versuche gibt, der Unterentwicklungstheorie durch die Analyse der internen Gesellschaftsstrukturen (Ungleichzeitigkeit der Instanzen Ökonomie, Politik und Ideologie als Ausdruck der Kombination verschiedener Produktionsweisen) neue Wege zu weisen (Schweers 1980, Senghaas 1982, Eikenberg 1983), stützt sich Immanuel Wallersteins Weltsystemtheorie erneut auf die Schlüsselbegriffe der Abhängigkeitstheorie, um mit ihrer Hilfe die weltgeschichtliche Ent wicklung seit dem 16. Jahrhundert zu erklären.

Wallerstein unternimmt den Versuch, das innere Band zwischen Spätfeudalismus und Kapitalismus, Handelskapital und Industriekapital, freier Lohnarbeit und zweiter Leibeigenschaft, Absolutismus und bürgerlichem Staat, Zentrum und Peripherie, Kapitalismus und Realsozialismus, Klassen, Nationalstaat und Weltsystem unter Anwendung eines theoretischen Begriffs, der kapitalistischen Weltwirtschaft, aufzuzeigen.

Wallersteins Kernthesen lassen sich kurz wie folgt zusammenfassen: Im 16. Jahrhundert ist ein historisch qualitativ neues Weltsystem entstanden. An die Stelle der redistributiven vorkapitalistischen Weltreiche, die den Surplus mit Hilfe einer Zentralinstanz politisch umverteilten, ist die kapitalistische Weltwirtschaft getreten, die auf der Grundlage einer internationalen Arbeitsteilung, aber mebrever politischer Einheiten (Nationalstaaten) die weltweite Aneignung des Surplus organisiert. Das neue Weltsystem gliedert sich in Zentrum, Semiperipherie und Peripherie, drei Subsysteme, die über den Mechanismus des ungleichen Tausches verknüpft sind. Da das internationale Arbeitsteilungssystem von einer Produktionsweise, der kapitalistischen, dominiert wird, sind auch die Arbeitssysteme der Peripherie die zweite Leibeigenschaft in Osteuropa, die Formen ungleicher Arbeit in den Kolonien (encomienda, Sklaverei) - sowie das System des Staatseigentums an den Produktionsmitteln in den semiperipheren »realsozialistischen « Staaten nicht als vorkapitalistische oder nacbkapitalistische Produktionsverhältnisse zu interpretieren; sie sind dem Arbeitsteilungs- und 
Ausbeutungsnexus der kapitalistischen Weltökonomie unterworfen und üben innerhalb dieses Systems besondere Funktionen aus.

Das Weltsystem kennt seit dem 16. Jahrhundert zwei Zeitalter, das des Agrar- und Industriekapitalismus, und vier Entwicklungsphasen: Die Grundlegung des modernen Systems (Phase I: 1450-1640), die Konsolidierung der neuen kapitalistischen Weltwirtschaft in der Krise des 17. Jahrhunderts (Phase II: 1640-1730), die erste und zweite Phase des Industriekapitalismus, deren Zäsur die Revolution in Rußland (1917) bildet (Phase III und IV). Das kapitalistische Weltsystem wird seit Anbeginn von drei zentralen Widersprüchen beherrscht, dem Klassenkampf zwischen Lohnarbeit und Kapital, dem Krisenzyklus, der aus der Überproduktionstendenz des Weltkapitals resultiert, sowie der Ökonomie/Politik-Antinomie, die Ausdruck der Diskrepanz zwischen weltweiter ökonomischer Einheit und nationalstaatlicher Vielfalt ist.

Die bisherige kritische Auseinandersetzung mit dem Weltsystemansatz Wallersteins ist sehr stark von Historikern getragen worden. Da sich Wallersteins Analyse der kapitalistischen Weltwirtschaft bisher auf die ersten beiden Phasen beschränkt, standen Fragen des Überganges vom Feudalismus zum Kapitalismus, des Verhältnisses von Handels- und Industriekapital, des Unterschiedes zwischen Merkantilismus und Industriekapitalismus, des Klassencharakters des Absolutismus, der Bestimmung der Produktionsverhältnisse in Osteuropa und in den Kolonien im Vordergrund der Debattte. Mir geht es in diesem Beitrag darum, die zentralen theoretischen Begriffe der Weltsystemtheorie Wallersteins zu hinterfragen. Die Kategorie "kapitalistische Weltwirtschaft", deren Inhalt Wallerstein der Dependenztheorie entliehen hat, ist in der Tat der Eckstein seiner fünf Jahrhunderte und drei Gesellschaftssysteme umspannenden Theorie. Ich werde Wallersteins Bestimmung des Verhältnisses von Nationalstaat und Weltmarkt, seine Theorie der internationalen Arbeitsteilung, seine Erklärung des Positionswechsels zwischen den drei Subsystemen (Zentrum, Semiperipherie, Peripherie) sowie seine Hegemoniezyklustheorie näher beleuchten; danach werde ich neuere theoretische Ansätze zur Reformulierung der Unterentwicklungsproblematik vorstellen, um abschließend die wissenssoziologische Frage nach der Zählebigkeit der Dependenztheorie zu diskutieren, die m.E. zentral mit dem politischen Bedürfnis der Linken nach einer Theorie des internationalen Klassenkampfs zu tun hat.

\section{Das Verhåltnis von Weltmarkt und Nationalstaat}

In der bisherigen Geschichte gibt es nach Wallerstein zwei Arten von Weltsystemen: "Weltreiche« und "Weltwirtschaften«。 "Logisch gefolgert, kann es freilich zwei Spielarten solcher Weltsysteme geben: Ein Weltsystem mit einem gemeinsamen politischen System und ein Weltsystem ohne gemeinsames politisches System. Wir wollen die erste Variante mit dem Begriff 'Weltreiche', die zweite mit dem Begriff 'Weltwirtschaften' kennzeichnen " (Wallerstein $1979 \mathrm{~b}, 35)$.

Beide Formen von Weltsystemen stützten sich auf eine internationale Arbeitsteilung, die deren Einheit konstituiere. Während jedoch in den Weltreichen der Surplus über redistributive Methoden verteilt worden sei, übernehme in der Weltwirtschaft der Markt die Verteilungsfunktion. "Erst mit der Herausbildung der modernen Weltwirtschaft in Europa des 16. Jahrhunderts kam es zur vollen Entfaltung und ökonomischen Vorherrschaft des 'Markthandels' - zu der Errichtung des Systems, das 'Kapitalismus' heißt. Kapitalismus und Weltwirtschaft 
(d.h. eine einzige Arbeitsteilung, aber vielfältige Gemeinwesen und Kulturen) sind zwei Seiten ein und derselben Medaille« (Wallerstein 1979b, 35 f.; vgl. auch: Wallerstein 1979a, 159).

Wallerstein hält es für systembedingt, daß der Kapitalismus sich nicht innerhalb eines Weltreiches, sondern im Rahmen einer Weltwirtschaft mit einer ökonomischen Arbeitsteilung, aber mehreren Nationalstaaten entwickelt habe. Das Auseinanderfallen von ökonomischer und politischer Einheit erlaube dem Kapital, im Sinne seiner ökonomischen Interessen auf die Staaten Eimfluß zu nehmen. Im Rahmen eines einzigen Weltstaates wäre dies nicht möglich, weil dessen Regierungen vielfältigen Interessen und Einflußnahmen zu genügen hätten. »Aber der Kapitalismus kann nicht im Rahmen eines Weltreiches florieren. Dies ist ein Grund, weshalb er sich nicht im Römischen Reich entwickelte. Die verschiedenen Vorteile, über die das Handelskapital in der entstehenden Weltwirtschaft verfïgte, könnten innerhalb eines einzigen Staates, dessen Regierungen dem Druck verschiedener Interessen ausgesetzt wären, politisch nicht so leicht durchgesetzt werden«(Wallerstein 1974, 127; vgl. auch; Wallerstein 1980, 113)."

An einer anderen Stelle wird diese These näher begründet: $\gg$ Der Kapitalismus als ein Produktionssystem des Markthandels zum Zwecke der Profitaneignung auf der Grundlage privaten oder kollektiven Eigentums hat nur in einem Weltsystem existiert, in welchem die politischen Einheiten nicht mit den Grenzen der Marktökonomie zusammenfallen. Dies hat den Händlern erlaubt, von bestehenden Marktvorteilen - wann immer es sie gab - zu profitieren, sie aber gleichzeitig befähigt - wann immer es notwendig war -, Druck auf die politischen Instanzen auszuüben, zu ihrem Vorteil regulierend in den Markt einzugreifen « (Wallerstein 1979a, 66; vergl. auch: $69 \mathrm{f}_{\text {. }}$ ).

Diese Argumentation Wallersteins zum Verhältnis von Weltwirtschaft und Nationalstaat ist kritikbedürftig. Seine These impliziert eine problematische Bestimmung des Verhältnisses der Instanzen Politik und Ökonomie innerhalb der kapitalistischen Produktionsweise. Er hält die Annahme freier, d.h. staatsfreier ökonomischer Konkurrenz für eine ideologische Doktrin und behauptet: »...die Kapitalisten versuchen, ihren Profit, den sie auf dem Weltmarkt erzielen können, zu maximieren. Dabei organisieren sie, wann immer es profitabel ist und wann immer sie dazu fähig sind, legale Monopole und/oder andere Formen von Handelsbeschränkungen «(Wallerstein 1979a, 149).

Mit dieser Prämisse übersieht Wallerstein die spezifische Differenz zwischen der kapitalistischen und den vorkapitalistischen Produktionsweisen. Die Entschränkung der politischen und der ökonomischen Instanz ist gerade das Kennzeichen einer Produktionsweise, in der die Aneignung des Mehrprodukts des direkten Zwangseingriffs nicht mehr bedarf. Die sich ökonomisch vermittelnde Ausbeutung der Ware Arbeitskraft durch das Kapital ermöglicht die staatliche Abstinenz von einer direkten Intervention in den ökonomischen Konkurrenzkampf. Die politische Instanz wird von der ökonomischen separiert und übernimmt als außerökonomische Zwangsgewalt die institutionelle Garantie der kapitalistischen Produktionsverhältnisse.

Nur auf der Grundlage dieses Verständnisses der unterschiedlichen Relationen zwischen den Instanzen Politik und Ökonomic in den vorkapitalistischen und in der kapitalistischen Produkrionsweise ist es verständlich, daß die bürgerlichen Revolutionen die merkantilistische Wirtschaftspolitik der feudalen absolutistischen Staaten nach innen destruieren, den einheitlichen Binnenmarkt, einheitliche Zölle, ein Währungsgebiet sowie die Gewerbefreiheit einführen. Und nur auf diesem Hintergrund läßt sich die Frage nach dem Verhältnis von kapita-

* Alle Zitate englischsprachiger Titel sind von mir, K.B., übersetzt. 
listischer Weltwirtschaft und dem System der Nationalstaaten sinnvoll stellen. Die Differenz zwischen den nationalen Binnenmärkten und den nationalstaatlich strukturierten Weltmärkten ist in der unterschiedlichen Präsenz der Instanz Politik im Konkurrenzkampf der Kapitale zu sehen. Während auf den narionalen Binnenmärkten der Konkurrenzkampf sich auf der Grundlage primär ökonomischer Mechanismen (Ausbeutungsrate, Produktivität und Intensität der Arbeit) ohne staatliche Bevorzugung von Einzelkapitalen vollzieht, interveniert der Nationalstaat in den internationalen Konkurrenzkampf des Kapitals durch Zölle, Importbeschränkungen, Währungsparitätsveränderungen etc. direkt zugunsten von einzelnen nationalen Kapitalen. Das Problem der Beziehung zwischen den Instanzen Politik und Ökonomie ist darin zu sehen, daß die bürgerlichen Revolutionen im Inneren der neuen Gesellschaften die Logik der kapitalistischen Produktionsweise, d.h. die Separierung von Politik und Ökonomie, durchgeserzt, nach außen aber die vorkapitalistischen Strukturen, d.h. die Möglichkeit direkter staatlicher Intervention in die Ökonomie, beibehalten haben. Wallerstein versperrt sich durch die mangelnde theoretische Abgrenzung der kapitalistischen von der feudalen Produ cions Weltmarkttheorie. Es 1.t an anderer Stelle (Busch/Grunert/Tobergte 1984, 25 ff.) begründet worden, unter welchen Bedingungen (Depressionsphase des Weltkapitals) der bürgerliche Nationalstaat auf sein Interventionspotential für den internationalen Konkurrenzkampf des Kapitals zurückgreift und unter welchen Voraussetzungen (Aufschwungsperioden des Weltkapitals) er sich auf ein ihm eigenes Interventionsminimum (Währungsmechanismen) zurückzieht. Hier sei nur angemerkt, daß Wallerstein auf der Grundlage seiner methodischen Prämissen nicht in der Lage ist, die besonderen ökonomischen Gesetzmäßigkeiten zu erkennen, die den Weltmarkt in Phasen günstiger Kapitalakkumulation (Liberalisierungsphasen) prägen. Dieser erste „Sündenfall« Wallersteins bleibt nicht ohne Folgen: Er beherrscht die Prämissen seiner Theorie der internationalen Arbeitsteilung und seiner Theorie der ungleichmäßigen Entwicklung ${ }^{1}$.

\section{Die Struktur der Arbeitsteilung in der kapitalistischen Weltwirtschaft}

Wallerstein begreift die kapitalistische Weltökonomie als System. Unter Berufung auf Dependenztheoretiker wie Baran, Frank, Amin und Emmanuel grenzt er seine »world-system perspective« von der »developmentalist perspective« der Modernisierungstheorie (z.B. Rostow) ab. Während die Modernisierungstheorie einer »mechanistischen Methode« verpflichtet sei, denke der Weltsystemansatz in dialektischen Begriffen (Wallerstein 1979a, 52 ff.). »Ich verstehe unter diesem Begriff, daß die Analyse in jedem Schritt nicht nach der formalen Struktur, sondern nach den Folgen für das Ganze und seine Teile fragen soll, wenn eine bestimmte Struktur bewahrt oder verändert wird ... «(Wallerstein 1979a, 54). Abgesehen von dem äußerst unterentwickelten Dialektikverständnis, das hier präsentiert wird (vergl. Althusser 1968), werden wir im folgenden sehen, daß Wallersteins Methode sich entgegen seiner Referenzen an »die Dialektik « als schlichter Systemformalismus erweist. Seine Begründung der Struktur der Arbeitsteilung innerhalb der kapitalistischen Weltökonomie lautet: »Wenn die Weltwirtschaft mit einer einzigen Arbeitsteilung die ökonomische Einheit darstellt, ist es natürlich, daß unterschiedliche Regionen unterschiedliche ökonomische Aufgaben wahrnehmen. Unter den Bedingungen des Kapitalismus ist es ohnehin natürlich, daß wir vom Zentrum, von der Peripherie und der Semiperipherie der W/ eltwirtschaft sprechen. Diese re- 
flektieren, da die politischen Grenzen (Staaten) enger sind als das ökonomische Ganze, unterschiedliche Gruppierungen ökonomischer Stärke und Aufgaben auf dem Weltmarkt« (Wallerstein 1979a, 60). Weiter heißt es: „Daß sich definitionsgemäß (Hervorh. i.O.) nicht alle Staaten gleichzeitig 'entwickeln' können, ist das wesentliche Moment der kapitalistischen Weltwirtschaft, denn das System funktioniert auf der Grundlage ungleich entwickelter Zentral- und Peripherieregionen « (Wallerstein 1979a, 61).

Per Definition wird hier eine ungleiche Arbeitsteilung zwischen Zentrum, Semiperipherie und Peripherie gesetzt, diese als im Kontext der kapitalistischen Weltökonomie »natürlich« bezeichnet und die Entwicklung innerhalb der kapitalistischen Weltwirtschaft als Null-SummenSpiel deklariert. Systemfunktional weist die Welteinheit ihren Teilen, den Nationalstaaten, unterschiedliche ökonomische Aufgaben zu. Wallerstein liefert hier formale, teilweise zirkuläre Definitionen, er argumentiert funktionalistisch und läßt Begründungen vermissen.

Neben diesem systemfunktionalistischen Argumentationsmuster lassen sich in Wallersteins Texten auch »materialere« Begründungen für sein Arbeitsteilungsmodell finden, deren Stringenz es im weiteren zu überprïfen gilt.

Innerhalb der kapitalistischen Weltökonomie unterscheidet Wallerstein drei Positionen: Zentrum, Semiperipherie und Peripherie. Die Besetzung dieser Positionen durch die verschiedenen Länder der Weltökonomie ergibt sich aus der Stärke der nationalen Staatsapparate, die wiederum auf der Konvergenz bzw. Divergenz der Interessen der herrschenden Klassen basiert. Gegenüber den Weltreichen, die primitive Formen ökonomischer Beherrschung sind, hat die kapitalistische Weltökonomie nach Wallerstein die »Technologie« erfunden, ohne eine beschwerliche und verschwenderische politische Superstruktur den Transfer von Surplus von der Peripherie ins Zentrum zu erhöhen (Wallerstein 1974, 15 f.). "Sobald es einen Unterschied in der Stärke der Staatsapparate gibt, beginnt auch der Mechanismus des 'ungleichen Tauschs' zu wirken, ein Mechanismus, den starke Staaten gegen schwache, Länder des Zentrums gegen periphere Gebiete einsetzen. Kapitalismus bedeutet also nicht nur, daß die Produktionsmittelbesitzer sich von denen, die nichts anderes als ihre Arbeitskraft besitzen, den Mehrwert aneignen, sondern Kapitalismus heißt auch die Aneignung des volkswirtschaftlichen Überschusses (Surplus) der gesainten Weltwirtschaft durch die Länder des Zentrums. Und dies galt für das Stadium des Agrarkapitalismus ebenso, wie es für das Stadium des Industriekapitalismus gilt«(Wallerstein 1979, 47; vgl. auch Wallerstein 1984, 13 ff.).

Im Unterschied zum oben kritisierten systemfunktionalistischen Argumentationsmuster, in dem das Weltsystem den Nationalstaaten ungleiche Positionen in der internationalen Arbeitsteilung zuwies, versucht $\mathbb{W}$ allerstein hier, den Mechanismus des ungleichen Tausches in der Weltökonomie zu begründen. Die unterschiedliche Stärke der Nationalstaaten, ihre ungleiche Fähigkeit, politisch in den internationalen Markt zu intervenieren, ist der Springpunkt des ungleichen Tauschs. Dem Zentrum gelingt es, aufgrund seiner politischen Stärke den internationalen Handel zu manipulieren, um sich so einen Teil des Surplus' der Peripherie anzueignen. Dieses Modell ungleicher Arbeitsteilung ist ohne Frage geeignet, die Struktur der Weltwirtschaft vom 16. bis zum 18. Jahrhundert zu erklären, als der internationale Handel weitgehend über politische und militärische Mechanismen sich vermittelte. Die merkantilistische Doktrin ist der Kulminationspunkt dieser von feudalen Prinzipien dominierten Weltwirtschaftsstruktur. A.ber Wallerstein begnügt sich nicht damit, seine Arbeitsteilungstheorie auf diese Raub- und Plünderungsphase der Weltwirtschaft zu begrenzen, er stilisiert dieses Modell des ungleichen Tausches zum allgemeinen Funktionsprinzip kapitalistischer Weltökonomie. Ehe ich diese problematische These Wallersteins näher beleuchte, möchte 
ich einen weiteren Begründungsversuch Wallersteins für die ungleiche internationale Arbeitsteilung vorstellen, der neben der obengenannten Argumentation in seinen Texten zu finden und mit dieser logisch nicht kompatibel ist. Der herrschenden Lehre in der neomarxistischen Dependenztheorie der letzten 15 Jahre folgend, greift auch Wallerstein auf Emmanuels Theorie des ungleichen Tauschs zurück, zitiert dessen Theorie an zahlreichen Stellen seiner Texte zustimmend (z.B. Wallerstein 1979a, 66 ff.; Wallerstein 1980, 92 ff.). Emmanuel begründet seine Theorie des ungleichen Tauschs allerdings nicht mit der unterschiedlichen Stärke der Nationalstaaten in der kapitalistischen Weltökonomie, sondern im Kern mit ökonomischen Mechanismen. Die Durchsetzung einer internationalen Durchschnittsprofitrate im Handel zwischen zwei Weltregionen, die sich bei gleicher technischer Zusammensetzung des Kapitals in ihrer Lohnhöhe fundamental unterscheiden, führt nach Emmanuel zu einem permanenten Mehrwerttransfer von den ökonomisch schwach entwickelten Ländern in die kapitalistischen Metropolen. Die Surplus-Aneignung durch die Zentren wird hier auf der Basis eines freien internationalen Handels mit freier Kapitalmobilität und eingeschränkter Arbeitskraftmobilität erklärt, also ökonomisch abgeleitet, während Wallerstein mit seiner $\mathrm{Hy}$ pothese von der ungleichen nationalstaatlichen Interventionsmacht in den internationalen Konkurrenzkampf den ungleichen Tausch über die Restriktionen des internationalen Handels (Monopole, constraint of trade) politisch begründet.

Indem Wallerstein mit beiden Modellen des ungleichen Tausches innerhalb seiner Weltsystemtheorie argumentiert, arbeitet er mit zwei sich logisch widersprechenden Prämissen. Sein Weltarbeitsteilungsmodell hält damit der Konsistenzprüfung nicht stand. Wir werden weiter unten sehen, daß sich diese Inkonsistenz der Theorie an entscheidenden Knotenpunkten der Wallersteinschen Argumentation als Fallgrube erweist.

Meine Kritik der Arbeitsteilungstheorie Wallersteins soll aber nicht bei dieser Konsistenzprüfung verharren, vielmehr möchte ich im folgenden zeigen, daß sich weder auf der Basis des politisch begründeten ungleichen Tausches noch auf der Grundlage des Emmanuelschen Modells eine begriffliche konsistente und empirisch gehaltvolle Weltarbeitsteilungstheorie formulieren läßt, also auch die Eliminierung des oben dargestellten logischen Widerspruchs der Weltsystemtheorie keine neue Perspektive bietet.

Ich habe bereits darauf hingewiesen, daß Wallerstein die Entwicklung innerhalb der Weltwirtschaft als Null-Summen-Spiel betrachtet: Gewinne einiger Länder sind systembedingt mit Verlusten anderer Länder verbunden: "Um es konkret zu formulieren: es ist theoretisch nicht möglich, daß sich alle Staaten gleichzeitig 'entwickeln'. Die sogenannte 'wachsende Lücke' ist keine Anomalie, sondern ein konstanter Grundmechanismus der Funktionsweise der Weltwirtschaft. Natürlich können sich einige (Hervorh. i.O.) Länder 'entwickeln', aber dieser Aufstieg geht nur zu Lasten anderer, die absteigen« (Wallerstein 1979a, 73).

Diese Null-Summen-Hypothese ist Resultat der Wallersteinschen Analyse des internationalen Konkurrenzkampfs des Kapitals. Da die Staatsmaschinerien von den jeweiligen nationalen Kapitalgruppen stets dazu eingesetzt werden, sich Vorteile auf Kosten anderer Nationalkapitale $z u$ verschaffen, muß die Gewinn- und Verlust-Bilanz im Gesamtsystem ausgeglichen sein. »Die Staatsapparate haben seit Beginn des Kapitalismus in den Weltmarkt eingegriffen. Mehr noch, die Staaten haben sich - jeder in Beziehung zu anderen - gebildet, entwickelt und militarisiert, um so die Verteilung des Mehrwerts beeinflussen zu können« (Wallerstein 1979a, 274).

Der Mangel dieser theoretischen Position besteht darin, daß nicht nur die merkantilistische Expansionsperiode feudaler Staaten mit der außenwirtschaftlichen Expansionspolitik kapi- 
talistischer Staaten konfundiert, sondern auch keine Unterscheidung zwischen liberalistischen und protektionistischen Phasen kapitalistischer Weltmarktexpansion vorgenommen wird. Wallerstein kann damit nicht mehr erklären, unter welchen Voraussetzungen der $\mathrm{Na}$ tionalstaat sich direkter Interventionen in den internationalen Konkurrenzkampf des Kapitals enthält und welche $Z$ wänge ihn zu einer protektionistisch-merkantilistischen Politik veranlassen. Wallerstein zufolge dient der Liberalismus nur den Interessen der Hegemonialmacht, bereits die zweitplazierten Mächte des Weltsystems sind auf den Merkantilismus angewiesen (Wallerstein 1979b, 48; vgl. auch Wallerstein 1984, 37 ff.). Wie läßt sich aber mit diesem Denkansatz etwa die Phase des Liberalismus von 1950 bis 1975 erklären, in der nicht nur die USA, sondern gerade auch ihre schärfsten (zweit- und drittplazierten) Konkurrenten in Westeuropa und Japan vom Freihandel profitierten? Das auf der unterschiedlichen Stärke der Nationalstaaten fußende Konzept des ungleichen T'auschs versagt hier vollkommen: Diejenige Macht, die in ökonomischer, politischer und militärischer Hinsicht über eine absolute Hegemonialposition verfügte, die USA, verlor in einer Phase des Liberalismus (!) relativ an Terrain zugunsten Westeuropas und Japans. Offensichtlich geht Wallersteins Weltsystemtheorie von einer falschen Prämisse aus. Auf der Basis des Freihandels führt der Konkurrenzkampf des Kapitals auf dem Weltmarkt entgegen Wallersteins Dependenzhypothese zu einer Arbeitsteilung, von der auch die ökonomisch schwächer entwickelten Nationalkapitale profitieren. Der internationale Konkurrenzkampf des Kapitals ist kein Null-Summen-Spiel, sondern führt zu einer Arbeitsteilung auf der Grundlage komparativer Kostenvorteile, die dem Gesamtsystem und seinen Teilen Produktivitätszuwächse ermöglicht. Schwächer entwickelte Länder können wegen der Schutzmechanismen der modifizierten Wirkungsweise des Wertgesetzes auf dem Weltmarkt ihre spezifischen Akkumulationsvorteile(höhere Durchschnittsprofitrate) ausschöpfen und sogar höhere Wachstumsraten als die Zentren des Weltkapitals realisieren (vgl. dazu: Busch 1974 und 1978). Wallerstein ist nicht in der Lage, diese besonderen Gesetzmäßigkeiten der Kapitalkonkurrenz auf dem Weltmarkt zu entschlüsseln, weil er sich bereits in der Bestimmung des Verhältnisses von Politik und Ökonomie im Weltsystem die Erkenntnis der Struktur der kapitalistischen Produktionsweise verschüttet. In diesem ersten fundamentalen theoretischen Mangel wurzeln alle weiteren Fehlannahmen Wallersteins. Theorien dagegen, die von der Separierung der Instanzen Politik und Ökonomie als Grundstruktur der kapitalistischen Produktionsweise ausgehen, sind in der Lage, (a) die Besonderheiten des Nationalstaates in der kapitalistischen Weltökonomie und (b) die aus dieser Nationalstaatsstruktur resultierende modifizierte Form der Kapitalkonkurrenz auf dem Weltmarkt zu erkennen².

Wie auch viele andere Dependenztheoretiker (Amin, Frank, Senghaas) stützt sich Immanuel Wallerstein in seinem Weltsystemansatz darüber hinaus auf einen zweiten Begriff des ungleichen Tauschs, denjenigen Emmanuels (Emmanuel 1972). $\gg$ Der Austausch zwischen Zentrum und Peripherie eines kapitalistischen Systems, der aus dem Tausch von Hochlohngegen Niedriglohnprodukte besteht, impliziert einen 'ungleichen Tausch' im Sinne Emmanuels, wonach bei einem gegebenen Produktivitätsniveau der Arbeiter der Peripherie mehrere Stunden arbeiten muß, um ein Produkt zu erwerben, das der Arbeiter des Zentrums in einer Stunde erzeugt hat. Und umgekehrt. Solch ein System ist für die Expansion des Weltmarktes notwendig (Hervorh. i.O.), wenn der Profit (Hervorh。 i.O.) das primäre Ziel darstellt. Ohne den ungleichen (Hervorh. i.O.) Tausch wäre es nicht profitabel (Hervorh. i.O.), die Arbeitsteilung auszudehnen. Und ohne eine solche Expansion wäre es nicht profitabel, die kapitalistische Weltwirtschait aufrechtzuerhalten. Eine Desintegration der kapitalisti- 
schen Weltwirtschaft oder die Rückkehr zu Formen eines redistributiven Weltreiches wären die Folge«(Wallerstein 1979a, 71).

Emmanuel leitet in seinem Hauptwerk den ungleichen Tausch aus drei grundlegenden Prämissen ab:

1. Die internationale Kapitalmobilität führt zu einer internationalen Durchschnittsprofitrate auf der Basis internationaler Produktionspreise.

2. Die internationale Immobilität der Ware Arbeitskraft drïckt sich in ungleichen nationalen Lohnniveaus aus.

3. Der Lohn ist in bezug auf den Preis die unabhängige Variable.

Die Nationen, in denen es der Arbeitskraft gelungen ist, durch den gewerkschaftlichen Kampf das Lohnniveau zu heben, seien in der Lage, die Kapitalakkumulation zu beschleunigen. Denn hohe Löhne implizieren a) eine Steigerung der organischen Zusammensetzung des Kapitals, also eine Entwicklung der Produktivkräfte, b) eine Realisierungsbasis für die erweiterte Reproduktion des Kapitals (Nachfragemoment) und c) einen permanenten ungleichen Tausch im internationalen Handel zugunsten des entwickelten Landes. Entwicklung stellt sich daher nicht als Ursache, sondern als Folge hoher Löhne dar - Reichtum ruft Reichtum hervor, Armut Armut.

Diese Theorie ist aus zwei Gründen, einem empirischen und einem theoretischen, auf starken Widerstand gestoßen (Busch 1973; Schweers 1980). Empirisch geht Emmanuel von einem so hohen internationalen Kapitalfluß zwischen den armen und den reichen Ländern aus, daß sich real eine Durchschnittsprofitrate zwischen diesen beiden Weltregionen konstituiert. Angesichts der relativ (d.h. sowohl in bezug auf die gesamten Auslandsinvestitionen als auch in bezug auf das gesamte Investitionsvolumen in der kapitalistischen Welt) geringen Bedeutung der Direktinvestitionen der entwickelten Industrieländer in den Ländern der $»$ Dritten Welt « ist diese Annahme aber entschieden zu bestreiten. Darüber hinaus impliziert diese Prämisse Emmanuels eine Paradoxie: Wäre der Kapitalfluß in die »Dritte Welt « so immens, daß wir berechtigt von einer Weltdurchschnittsprofitrate sprechen könnten, müßten sich die Produktivkräfte in der Peripherie rasant entfalten, würde die Unterentwicklung durch einen massiven Transfer von Produktionsstätten aus den Metropolen in die "Dritte Welt» überwunden werden.

Theoretisch sieht Emmanuel in den internationalen Produktionspreisen die Form der Durchsetzung einer allgemeinen internationalen Profitrate. Er negiert dabei die spezifischen Momente der modifizierten Wirkungsweise des Wertgesetzes auf dem Weltmarkt, die zu der Annahme berechtigen, daß der internationale Profitratenannäherungsprozeß sich gerade nicht im Medium internationaler Produktionspreise, sondern über einen branchenspezifischen Profitratendifferenzierungsprozeß auf Weltebene vollzieht (Busch 1974, 46 ff.).

Emmanuels Modell erweist sich damit als empirisch irrelevant und theoretisch inkonsistent. Auch diese Variante des ungleichen Tauschs kann damit nicht als der Eckstein betrachtet werden, der die Null-Summen-Optik des Weltsystemansatzes zu stützen vermag.

\section{Positionsveränderungen innerhalb des Weltsystems - Über die Chance semiperipherer Staaten, ins Zentrum aufzusteigen}

In seinen Abhandlungen zur Struktur der kapitalistischen Weltökonomie reflektiert Wallerstein auch die Frage der Transformationsmöglichkeiten innerhalb des Weltsystems, der Chancen zum Positionswechsel von Staaten zwischen den Subsystemen Zentrum, Semiperi- 
pherie und Peripherie. Dieses Problem ist im Rahmen der Wallersteinschen Theorie von besonderem Interesse, müßte doch seine Arbeitsteilungstheorie (Null-Summen-Hypothese, ungleicher Tausch) der Transformationskapazität des Weltsystems sehr enge Grenzen ziehen. Während Wallerstein in seinen beiden Schriften zum »Modern World-System « diese Frage theoretisch nur streift (Wallerstein 1974, $80 \mathrm{ff}$. und $218 \mathrm{ff}$.; Wallerstein 1980, $38 \mathrm{f}$. und $179 \mathrm{ff}$.), widmet er sich in zwei Aufsätzen seines Readers » The capitalist world-economy« diesem Problem am Beispiel der Aufstiegschancen semiperipherer Staaten in das Zentrum des Weltsystems.

Wallerstein formuliert den Untersuchungsgegenstand hier wiederum im Kontext der Lohnproblematik: »Um abschätzen zu können, zu welchem Grad die semiperipheren Länder die klassischen Mechanismen des Aufstiegs in der Weltwirtschaft nutzen können, müssen wir diesen klassischen Mechanismus und die Rolle der Lohndifferenzen bei der Strukturierung der Weltwirtschaft analysieren "(Wallerstein 1979a, 84). Etwas weiter heißt es im selben Text: »Emmanuel argumentiert, daß die relative Starrheit der nationalen Lohnniveaus und die Tendenz zum Ausgleich der internationalen Profitraten für den ungleichen Tausch in der Weltwirtschaft verantwortlich sind. Dennoch ist es genau diese Starrheit, die den Wechsel semiperipherer Länder mit mittleren (Hervorh. i.O.) Lohnniveaus in die Gruppe der Zentrumsländer historisch möglich macht. Das Problem des Durchbruchs besteht für ein semiperipheres Land darin, daß es über einen Markt verfügen muß, der den Einsatz einer fortgeschrittenen Technologie, mit der es billiger produzieren kann als die bisherigen Anbieter, von der Größe her rechtfertigt« (Wallerstein 1979a, 84 f.).

Mit dieser Argumentation verwickelt sich Wallerstein in die Fallstricke seiner auf Emmanuel fußenden Theorie. Einerseits war das internationale Lohngefälle - wie wir oben sahen - die Begründung für die Ausbeutung der Niedriglohnländer (Mehrwerttransfer in das Zentrum), andererseits soll jetzt das niedrige Lohnniveau der Semiperipherie die Grundlage für eine günstige internationale Wettbewerbsposition und damit für einen Wechsel in den Metropolenstatus sein (vgl. auch: Wallerstein 1974, 81 ff. und 218 ff.). Die Behauptung, die Löhne seien die Basis der ungleichen Arbeitsteilung und gleichzeitig das Fundament für einen Aufstieg in der internationalen Hierarchie, stellt einen weiteren logischen Widerspruch innerhalb der Theorie Wallersteins dar. Die Inkompatibilität der Lohnkonkurrenzhypothese mit der Theorie des ungleichen Tausches kann anhand der weiteren Argumentation Wallersteins näher aufgezeigt werden. "Wenn hohe Löhne nach der Theorie des ungleichen Tausches so vorteilhaft sind, warum erhöht dann nicht jeder das Lohnniveau, zumindest jeder Staat? Weil offensichtlich der Vorteil auch eine Funktion der niedrigen absoluten Kosten ist (ganz abgesehen vom Preisniveau). Das Kapital fließt stets in die Bereiche mit den höchsten Profiten, aber es 'fließt', es besteht stets eine zeitliche Verzögerung. Wenn einige Produzenten in den Produktionskosten unterboten werden, besteht die Tendenz, eine neue Spezialisierung zu entdecken, die eine momentan seltene Fertigkeit erfordert und die in der interna-. tionalen Arbeitsteilung von den Niedriglohnländern zur Zeit nicht besetzt ist (Emmanuel 1972, p. 145)«(Wallerstein 1979a, 88).

Die These der internationalen Lohnkostenkonkurrenz wird also hier wieder eingeschränkt: Die Zentren des Weltkapitals entgehen der Lohnkonkurrenz durch eine Spezialisierung auf hochwertige neue Technologieprodukte, die die Niedriglohnländer noch nicht produzieren können. Spielen wir diese Modellannahme gedanklich durch, bleibt ebenfalls für die Ungleichheitshypothese kein Raum. Die Weltarbeitsteilung wäre in diesem Falle nach einem Lohn/Produktivitäts-Gefälle strukturiert: Länder mit niedrigerem Lohnniveau hätten in 
Branchen mit geringerem Technologieniveau Spezialisierungschancen, Hochlohnländer in Bereichen der Spitzentechnologie. Da nun in diesem Denkansatz die Schwellenländer in Sektoren mit mittlerem Lohn- und Produktivitätsniveau die entsprechenden Branchen der Zentren zurückdrängen würden, also sich hier überdurchschnittlich Marktanteile aneignen könnten, umgekehrt die Kapitale der Zentren überdurchschnittlich in Branchen mit Spitzentechnologie expandieren würden, wäre die Verteilung von Vor- und Nachteilen international ausgeglichen. Beide Weltregionen würden von der internationalen Arbeitsteilung profitieren; in der Tat handelt es sich hier um nichts anderes als die Theorie komparativer Kosten in der Begründung durch Ohlin und Heckscher. Weder die Theorie der direkten internationalen Lohnkostenkonkurrenz noch die Hypothese der internationalen Lohn/ Technologie-Konkurrenz kann die Theorie des ungleichen Tausches und damit der ungleichen internationalen Arbeitsteilung untermauern. Wallerstein scheint sich der Aporie seiner Argumentation über die Bedeutung der Löhne für den Wechsel von der Semiperipherie in das Zentrum bewußt zu sein. Da er der Behauptung, das mittlere Lohnniveau sei die Grundlage für den Aufstieg der semiperipheren Staaten, sogleich durch die These widerspricht, die Kapitale der Zentren könnten in technologieintensive Sektoren ausweichen und so im Sinne Emmanuels die Ungleichheit reproduzieren, versucht er, durch die Einführung einer neuen Variable seinen Überlegungen mehr Konsistenz zu verleihen. Die Berücksichtigung des Krisenzyklus des Weltkapitals erscheint Wallerstein als ein Ausweg aus seinem selbst erzeugten Labyrinth: "Ich glaube, daß den semiperipheren Ländern noch heute der Aufstieg ins Zentrum möglich ist, damit aber gleichzeitig die Perspektiven für die Peripherie verschlechtert werden. In Zeiten der Abschwächung der Weltkonjunktur wird das schwächste Segment der Weltwirtschaft als erstes in seiner Verhandlungsposition beeinträchtigt. Der relative Rückgang der Weltproduktion reduziert die Märkte für die Exporte der peripheren Länder ... In der Semiperipherie vollzieht sich dagegen ein anderer Prozeß. In einer expandierenden Weltwirtschaft sind die semiperipheren Länder Bettler, die die 'Hilfe' des Zentrums ersuchen, um einen Teil des Weltmarktes auf Kosten anderer semiperipherer Länder (Hervorh. i.O.) erobern zu können ... Während der Expansionsperiode vollzieht sich also die ökonomische Properität in der semiperipheren Region durch eine Verstärkung der Abhängigkeit vom Zentrum. In Abschwungsperioden dagegen sitzen die Länder des Zentrums, die fortfahren einander ökonomisch zu bekämpfen, weil jedes den Abstieg befürchtet, in der Klemme. In dieser Situation wird die Semiperipherie umworben, da die Absatzmärkte für Produkte des Zentrums relativ enger werden« (Wallerstein 1979a, 88 f.). Wallerstein vergleicht die verbesserte bargaining-Position der Semiperipherie in der Krise mit der stukturell günstigen Kampfposition der Leibeigenen gegenüber ihren Grundherren in der Krise ausgangs des Mittelalters. Den Krisenzyklus des Weltkapitals leitet Wallerstein wiederum aus dem Prozeß der weltweiten Surplus-Umverteilung zugunsten der Zentren ab (Wallerstein 1979a, $97 \mathrm{ff}$.). Die Surplus-Aneignung durch das Zentrum führe mittelfristig zu einer Kontraktion der Märkte für hochlohn- und technologieintensive Produkte, weil die Kaufkraft der ausgebeuteten Peripherie und Semiperipherie zurückgehe. Nun beginne die große Stunde einiger Staaten der Semiperipherie. Die Konkurrenz der core producers auf schrumpfenden Märkten lenke den Profitstrom in einige besonders starke semiperiphere Staaten um. Während es in den Staaten des Zentrums zu Massenarbeitslosigkeit komme, lege die Krise das Fundament für den Aufstieg einiger semiperipherer Staaten innerhalb der internationalen Hierarchie (vgl. dazu auch: Wallerstein 1979a, 62, 162, 275; Wallerstein 1980, $129 \mathrm{ff}.)^{3}$. 
Diese Argumentation Wallersteins weist m. E. eine Reihe eklatanter Mängel auf:

1. Wie wir oben gezeigt haben, hat Wallerstein keine konsistente Begründung für den Surplus-Transfer in die Zentren des Weltkapitals vorlegen können. Sein Weltüberproduktionszyklus, der aus der Surplus-Aneignung durch dieZentren resultiere, steht damit auf tönernen theoretischen Prämissen.

2. Konzedieren wir nun hypothetisch, es gäbe diesen Umverteilungsprozeß des internationalen Surplus;, ist uneinsichtig, weshalb die Semiperipherie von der Krise profitieren soll. Sie selbst, deren Absatzmärkte in den Zentren des Weltkapitals liegen, würde in den Abwärtssog der Überproduktionskrise hineingezogen werden. Ihre Importkapazität nähme wegen der schrumpfenden Exporte stark ab, die internationale bargaining power der Semiperipherie würde nicht wachsen, sondern zumindest ebenso geschwächt wie die der Zentren. (Vgl. das Problem der OPEC und der nichtölproduzierenden Schwellenländer in der Weltwirtschaftskrise 1981/83, insbesondere die Verschuldungskrise einiger Schwellenländer).

3. Nicht nachvollziehbar ist auch die Wallersteinsche Differenzierung zwischen der Semiperipherie und der Peripherie. Während diese aus der Krise geschwächt hervorgehen soll, profitiere jene aus einer gewachsenen Verhandlungsmacht. Wallersteins These, die Exporte der Peripherie stießen in der Krise auf höhere protektionistische Schutzwälle, gilt ebenso, ja sogar in größerem Maße für die Staaten der Semiperipherie. Da gerade die Schwellenländerexporte die Position einiger Branchen des Zentrums (Stahl, Textil, Schiffbau) bedrohen, richtet sich der »neue « Protektionismus der kapitalistischen Industrieländer gezielt gegen diese Länder, während den industriearmen peripheren Staaten gleichzeitig Handelszugeständnisse gemacht werden.

Auch Wallersteins Bemühung, mit Hilfe seiner Zyklustheorie den Positionswechsel innerhalb des Weltsystems zu begründen, ist also ein Fehlschlag. Auf der Grundlage seiner theoretischen Prämissen - Null-Summen-Hypothese, ungleicher Tausch -, ist das Aufsteigen schwächer entwickelter Länder in der internationalen Arbeitsteilungshierarchie nicht begri̊ndbar.

\section{Der Hegemoniezyklus der kapitalistischen Weltwirtschaft}

In seinem jüngsten Sammelband (Wallerstein 1984) legt Wallerstein u.a. auch einen theoretischen Rahmen für die in früheren Arbeiten (vor allem Wallerstein 1980, 38 ff.) nur verstreut zu findenden Anmerkungen zum Hegemoniezyklus im Weltsystem vor.

Hegemonie bedeutet die Fähigkeit einer Macht, als primus inter pares ihre Vorstellungen im ökonomischen, politischen, militärischen, diplomatischen und kulturellen Bereich des internationalen Systems durchzusetzen. Materielle Grundlage dieser Vorherrschaft sei ein Effizienzvorsprung der führenden Macht in Produktion, Handel und Finanzen: »Der Produktivitätsvorsprung, von dem wir sprechen, ist so groß, daß diese Unternehmen ihre Konkurrenten aus anderen Großmächten nicht nur auf dem Weltmarkt im allgemeinen ausstechen können, sondern in vielen Fällen insbesondere auf den Binnenmärkten der rivalisierenden Mächte selbst « (Wallerstein 1984, 39).

In der Geschichte des Weltsystems gab es nach Wallerstein drei Phasen, in denen eine Macht den Hegemoniestatus innehatte: Von 1620 bis 1672 (Holland), von 1815 bis 1873 (Großbritannien) und von 1945 bis 1967 (USA). In der Vorherrschaft dieser drei Mächte seien drei strukturelle Analogien auffällig: 
1. Der Erwerb und der Verlust ökonomischer Vorherrschaft habe sich von der Produktion über den Handel auf die Finanzen ausgedehnt.

2. Die Hegemonialmächte seien jeweils als Anwälte des ökonomischen und politischen Liberalismus und als Gegner des Merkantilismus aufgetreten.

3. Die Hegemonieposition sei jeweils nach einem Weltkrieg (Dreißigjähriger Krieg, Napoleonische Kriege, Kriege von 1914 bis 1945) erobert worden. Diese Weltkriege seien zeitlich dem Gipfel von langen Preiszyklen (»logistics«) zuzuordnen: dem Preiszyklus von 1450 bis 1730 mit 1600/1650 als Höhepunkt, dem Zyklus von 1730 bis 1897 mit 1810/17 als Gipfelpunkt und dem Zyklus von 1897 bis? mit bisher ungewissem oberen Wendepunkt.

"Wenn es solche Preiszyklen gibt, dann stellt sich heraus, daß der Weltkrieg und die darauf folgende Hegemonialära in etwa dem Gipfelpunkt (gerade davor und danach) des Preiszyklus zugeordnet sind. Das heißt, diese Prozesse scheinen das Produkt einer langen Konkurrenzphase zu sein, die zu einer besonderen Konzentration ökonomischer und politischer Macht geführt hat (Wallerstein 1984, 42). Das zyklische Hegemoniemuster innerhalb des internationalen Systems sei Ausdruck der Funktionsprinzipien der kapitalistischen Weltwirtschaft. Entgegen den Mythen vom freien Fluß der Produktionsfaktoren und von staatsfreier Konkurrenz auf den Märkten seien selektive politische Eingriffe des Staatsapparates für den Kapitalismus konstituriv. Der Hegemoniezyklus entstehe aus dieser Funktion des Staatsapparates in der kapitalistischen Weltökonomie. Das Kapital erstrebe mit Hilfe der nationalen Staatsapparate oligopolistische Positionen auf den internationalen Märkten. Um die ökonomischen Vorsprünge einer aufsteigenden Macht auf dem Weltmarkt fest zu verankern, werde der Krieg ins Spiel gebracht: "Die Vorsprünge, die die Unternehmen einer aufsteigenden Macht gegenüber ihren Konkurrenten aus vergleichbaren Mächten haben, dürften gering und von daher unsicher sein. Hier kommen die Weltkriege ins Spiel. Der Jahre dauernde Krieg mag militärisch und politisch sehr dramatisch sein, aber die tiefgreifendsten Folgen dürften ökonomischer Natur sein. Der ökonomische Vorsprung der Siegermacht wird durch den Krieg selber vergrößert, und das internationale Regime der Nachkriegszeit ist darauf angelegt, diesen größeren Vorsprung zu verfestigen und gegen Erosion zu schützen« (Wallerstein 1984,44$)$.

Die so eroberte Hegemonieposition währe aber aus zwei Gründen richt lange: Der von der Hegemonialmacht durchgesetzte Liberalismus trage zur Ausbreitung des technologischen Wissens der Vormacht innerhalb der gesamten Weltökonomie bei, und das Ansteigen der Realeinkommen der Lohnabhängigen der Hegemonialmacht verschlechtere deren Wettbewerbsposition auf dem Weltmarkt. Der Verlust der ökonomischen Vormachtstellung unterhöhle schließlich auch die politische Vorherrschaft der Hegemonialmacht und leite eine Phase der Neustrukturierung der Bündnissysteme innerhalb des Welrsystems ein.

Meine folgenden Ausfuihrungen werden sich ausschließlich mit Wallersteins Begründung des Hegemoniezyklus in der Weltökonomie beschäftigen; weitere kritische Einwände gegen seine hier dargestellten Thesen:

- Zum Verhältnis von Politik und Ökonomie im Kapitalismusbegriff,

- zur Konfusion von Spätfeudalismus und Kapitalismus beim Vergleich von Holland, England und den USA als Hegemonialmächten,

- zum Argumentationswiderspruch bei der Begründung des Verlustes der Hegemonieposition der Führungsmacht, die sich einerseits zu Lasten der übrigen Mächte bereichern soll 
(ungleicher Tausch über Monopolstatus), deren ökonomischer Vorsprung aber andererseits abgebaut werden kann,

- zum unzureichenden Verständnis liberaler Phasen der kapitalistischen Weltwirtschaft, ergeben sich implizit aus dem bisherigen Gang meiner Argumentation und müssen hier nicht wiederholt werden.

Wallerstein bettet den Hegemoniezyklus des politischen Systems in einen langen Preiszyklus (»logistic«) des ökonomischen Systems ein. Er legt allerdings keinerlei Erklärung für die Antriebskräfte des langen Preiszyklus vor. Ebensowenig reflektiert er die Frage, in welchem Zusammenhang der lange Preiszyklus evtl. mit langen Konjunkturzyklen (z.B. vom Typ Kondratieff) steht. Da unsere Kenntnis über lange Konjunkturwellen vor 1890 äußerst unzureichend sind (Kleinknecht 1984), sind Aussagen zur Zyklusproblematik vom 16. bis zum 19. Jahrhundert mit äußerster Vorsicht zu behandeln. Zum angeblichen Preiszyklus seit 1897 macht Wallerstein keine weiteren Ausführungen, obwohl unser Wissen über Wirtschaftszyklen im 20. Jahrhundert wesentlich gesicherter ist als über etwaige Zyklen in früheren Zeiten. Trotz dieser Kenntnislücken ordnet Wallerstein dem Höhepunkt der angeblichen drei langen Wirtschaftszyklen vom 16. bis zum 20. Jahrhundert drei verschiedene Kriegsereignisse zu und interpretiert diese als Hegemonialkriege. Dabei geht er so weit, den aufstrebenden Mächten Holland, Großbritannien und den USA jeweils eine direkte Auslöserrolle beim Dreißigjährigen Krieg, den Napoleonischen Kriegen und den beiden Weltkriegen des 20. Jahrhunderts zuzusprechen. Wallersteins Beobachtung, daß die politische und ökonomische Hegemonie häufig das Ergebnis weltweiter Kriege war, kann nicht bezweifelt werden. Zu hinterfragen aber ist seine Schlußfolgerung, daß die jeweils aufstrebende Macht deshalb ursächlich für diese Kriege verantwortlich sei. Auch die Interpretation der weltweiten Kriege im Zusammenhang mit bestimmten Phasen langer Wirtschaftszyklen ist äußerst problematisch. Dies wird etwa an den beiden Weltkriegen dieses Jahrhunderts deutlich, die - bei aller Unsicherheit über lange Zyklen - evtl. einem oberen Wendepunkt (Erster Weltkrieg) oder einem unteren Wendepunkt (Zweiter Weltkrieg) eines Kondratieff-Zyklus zugeordnet werden könnten. Wallersteins Verfahren, beide Weltkriege zusammenzufassen und sie dann noch einem nicht näher charakterisierten Preiszyklus zuzuordnen, ist vor diesem Hintergrund wissenschaftlich äußerst unseriös. Seine ökonomistische Analyse weltweiter Kriege im internationalen System reibt sich nicht an historischen Fakten, der Empirie stülpt sie ohne Umschweife ihr deterministisches Interpretationmuster über. Ohne hier näher die vier Kriegsperioden analysieren zu können, die Wallerstein anführt, ist evident, daß für diese Kriege neben ökonomischen Konflikten politische und ideologische Momente verantwortlich waren. Erst eine genauere theoretische Bestimmung des Verhältnisses der drei Instanzen Politik, Ökonomie und Ideologie im internationalen System, etwa im Sinne der Gesellschaftsanalyse Althussers, würde eine materialistische Analyse dieser Kriege ermöglichen. An die Stelle eines ökonomischen Determinismus - wie er für Wallersteins Analysen kennzeichnend ist - träte eine differenzierte Analyse der internationalen Gesellschaft, die die Dynamik der Ungleichzeirigkeit der Instanzen, des überdeterminierten Widerspruchs in den Mittelpunkt rückte.

Zusammenfassend möchte ich zur Frage des Hegemoniezyklus feststellen:

1. Unser Wissen über lange Wirtschaftszyklen vom 16. bis zum 20. Jahrhundert ist mit Ausnahme des laufenden Jahrhunderts sehr begrenzt.

2. Eine eindeutige Zuordnung von bestimmten Phasen eines langen Wirtschaftszyklus und Weltkriegen ist selbst im 20. Jahrhundert nicht möglich. 
3. Die Konstruktion eines Hegemoniezyklus, die Gipfelpunkte langer ökonomischer Preiszyklen als Ausbruchsphasen von Weltkriegen interpretiert, ist wissenschaftlich unhaltbar".

\section{Ansätze zur Reformulierung der Unterentwicklungsproblematik}

Die unterschiedlichen Entwicklungsprozesse, die sich in den Ländern Afrikas, Asiens und Lateinamerikas in den $70 \mathrm{er}$ Jahren trotz vergleichbarer Weltmarktbedingungen vollzogen haben, signalisieren die Krise der dependenztheoretischen Variante der Unterentwicklungsforschung. Offensichtlich läßt sich allein aus der Analyse der internationalen Handels- und Kapitalströme Unterentwicklung nicht konsistent erklären. In der notwendigen Neubesinnung der Unterentwicklungsfrage rücken deshalb jetzt die endogenen Probleme der ökonomisch schwach entwickelten Länder ins Zentrum des Interesses. Einige Resultate der neuen Forschungsanstrengungen sollen im folgenden vorgestellt werden.

Einer der Hauptvertreter der bundesrepublikanischen Dependenztheorie, Dieter Senghaas, ist aufgrund einer historischen Untersuchung nachholender Industrialisierungsprozesse in Europa zur Aufgabe des Dependenzparadigmas motiviert worden. Beruft Senghaas sich in früheren Arbeiten (Senghaas 1974, $30 \mathrm{ff}$. und 1977, $181 \mathrm{ff}$.) noch auf das Theorem des ungleichen Tausches in der Variante Emmanuels, heißt es nach der Untersuchung der Industrialisierungsgeschichte in Europa: "Wie die Darlegungen in diesem Kapitel aufgewiesen haben, wurden in den untersuchten Fällen, bei denen es sich nicht um typische fremdbestimmte Kolonialländer handelte, unter vergleichbaren Bedingungen des Weltmarktes die Weichen für autozentrierte Entwicklung bzw. Peripherisierung in den jeweiligen Gesellschaften selbst gestellt - und dieser Vorgang reflektierte unterschiedliche innergesellschaftliche Voraussetzungen für die Verbreitung von Chancen und Restriktionen, die vom Weltmarkt auf Entwicklungsprozesse einzelner Gesellschaften einwirkten ... Es ist daher ratsam, die Ursachen für autozentrierte Entwicklung oder Peripherisierung in den unterschiedlichen Transformations- und Innovationsfähigkeiten einzelner Gesellschaften zu suchen«(Senghaas 1982, 219).

Nicht mehr exogene, sondern endogene Faktoren bilden also den Fokus der Erklärung von Unterentwicklung. Allerdings hat Senghaas das dependenztheoretische Erbe nur halb überwunden. Zum einen fußen seine Reflexionen zur Akkumulation in ökonomisch schwach entwickelten Ländern weiterhin auf der problematischen Massenkaufkraftthese der lateinamerikanischen und französischen Abhängigkeirstheorie (vgl. dazu Schweers 1980), zum anderen hält Senghaas auch heute noch am Modell der autozentrierten Entwicklung fest, das sich stringent jedoch nur aus der Dependenztheorie begründen läßt (vgl. dazu Eikenberg 1983).

Theoretisch anspruchsvoller als Senghaas begründet Schweers in seiner Dissertation von 1979 die Notwendigkeit des Paradigmenwechsels in der Theorie der Unterentwicklung. Eine fundierte Untersuchung der Theorie und Empirie der Handels- und Kapitalbeziehungen zwischen den kapitalistischen Industrieländern und den Entwicklungsländern führt zu der Erkenntnis, »daß Unterentwicklung nicht logisch aus dem Wertgesetz und seiner Durchsetzung auf dem Weltmarkt zu erklären ist, sondern daß aufgrund der strukturellen Heterogenität in den unterentwickelten Ländern nur eine Theorie der Kapitalakkumulation sinnvoll erscheint, die die Ebene des idealen gesellschaftlichen Durchschnitrs verläßt und die Besonderheiten der unterentwickelten Länder systematisch auf die allgemeinen Gesetze der Kapi- 
talakkumulation bezieht. Da es also darum geht, den Grad der Entwicklung in Richtung auf das entfaltete Kapitalverhältnis zu bestimmen, muß die Vorgehensweise stärker bistorisch induktiv sein (Schweers 1980, 123 f.). Schweers lehnt die These ab, Unterentwicklung könne ausder Struktur des kapitalistischen Weltmarktes abgeleitet werden, auch das Massenkaufkrafttheorem der Dependenztheorie, das in dem Niedriglohnniveau der Entwicklungsländer eine prinzipielle Akkumulationsschranke sieht, hält er akkumulationstheoretisch für unausgewiesen. Eine historische Untersuchung des Industrialisierungsprozesses in England sowie in Preußen/Deutschland bestätigt diese Auffassung. Für das Gelingen sowohl der ursprünglichen Akkumulation als auch der nach holenden Industrialisierungsprozesse sind die endogenen Bedingungen kapitalistischer Akkumulation entscheidender als die exogenen. Die Frage Stagnation oder Entwicklung ist ohne eine Analyse der dominierenden Produktionsverhältnisse, der Klassenstrukturen und der Politik des Staatsapparates nicht zu beantworten.

Den theoretisch gehaltvollsten Versuch, die endogenen Bedingungen von Entwicklung bzw. Unterentwicklung zu formulieren, hat $\mathrm{m}$. E. Armin Eikenberg vorgelegt. Eikenberg zeigt die Bedeutung der Gesellschaftstheorie der Althusser-Schule für die Analyse des gesellschaftlichen Übergangs auf und reformuliert die Unterentwicklungsproblematik wie folgt: $\gg$ Das Verståndnis einer Gesellschaftformation als komplexe Totalität von ökonomischer, politischer und ideologischer Instanz vermeidet den Ökonomismus der Dependenztheorien und ermöglicht gleichzeitig, die Gesellschaftsformationen der Dritten Welt zu begreifen als konstituiert durch die Koexistenz verschiedener Produkrionsweisen, verschiedener politischer Strukturen und verschiedener Ideologien, wobei diese Koexistenz in unterschiedlichen Gesellschaftsformationen variiert, in unterschiedlichem Ausmaß instabil ist und sich im Ablauf von 'Zeit' verändert « (Eikenberg 1983; VIII f.).

Die Begriffe Produktionsweise, Gesellschaftsformation, gesellschaftliche ZEIT und gesellschaftlicher Übergang ermöglichen es, die Unterentwicklungsfrage als Problem der Ungleichzeitigkeit der Instanzen zu erfassen, als Koexistenzkampf ungleicher Reproduktionsrhythmen unterschiedlicher Produktionsweisen. Die ungleiche gesellschaftliche ZEIT der Instanzen Politik, Ökonomie und Ideologie sowie die instabile Koexistenz zwischen diesen Instanzen sind die zentralen Begriffe der »strukturalistischen Unterentwicklungstheorie. Homogenisierungen und Typisierungen des Gegenstandes lehnt Eikenberg ab, jedes Entwicklungsland ist auf seine spezifischen Bedingungen zur Überwindung von Unterentwicklung zu befragen.

Um eine Verknüpfung der weltmarktspezifischen und der gesellschaftsanalytischen Variablen einer Unterentwicklungstheorie bemüht sich ein Beitrag von Alain Lipietz (Lipietz 1984). Selbstkritisch verweist Lipietz auf den "pessimistischen Funktionalismus" der Dependenztheorie, die die Möglichkeit von Industrialisierungsmaßnahmen geleugnet habe. Er interpretiert die ökonomischen Differenzierungen im Zentrum und in der Peripherie als Ausdruck einer neuen internationalen Arbeitsteilung, die sich aus einer fordistischen Segmentierung des Produktionsprozesses nach Technologie- und Qualifikationsniveaus ergebe. Drei Segmente des Produktionsprozesses seien zu unterscheiden:

»i) Planung, Organisation und Konstruktion haben im wesentlichen einen autonomen Status erreicht;

ii) Produktion auf hohem technologischem Niveau, die qualifizierte Arbeitskräfte verlangt;

iii) Produktion auf geringem technologischem Niveau und Montage, die im wesentlichen an die Arbeitskräfte keine Qualifikationsanforderungen stellen« Lipietz 1984, 100). 
Die Konsequenzen für die internationale Arbeitsteilung beschreibt Lipietz wie folgt: "Es wurde dann möglich, diese drei Segmente auf drei geographische Regionen zu verteilen und den Produktionskreislauf der fordistischen Branchen auf drei Pole ungleich qualifizierter und ungleich bezahlter Arbeitskräfte auszudehnen. Diese regionale Reorganisation qualifizierter Arbeit war in den Zentren erprobt worden, während der sechziger Jahre wurden dann periphere Länder in unmittelbarer Nachbarschaft der Zentren einbezogen, deren Stundenlöhne beträchtlich niedriger und deren Gewerkschaften schwächer waren ...« (Lipietz 1984, 100).

Welche Stellung einzelne Länder in dieser neuen Form der internationalen Arbeitsteilung einnähmen, sei primär das Resultat interner Klassenkämpfe, die bestimmte Akkumulationsregime (regime of accumulation) und vom Staat organisierte Formen der gesellschaftlichen Regulation (mode of regulation) produzieren. In den Ländern der Peripherie gebe es zwei typische Schemata:

a) bloody taylorization: Produktionssegmente mit geringen Qualifikationsanforderungen an die Arbeitskräfte, hohe Ausbeutungsrate, Reexporte in die Zentren, z. B. Hongkong, Singapur;

b) peripheral fordism: Produktionssegmente mit hohen Qualifikationsanforderungen an die Arbeitskräfte, Binnenmarktentwicklung sowie Weltmarktexpansion im Bereich industrieller Fertigwaren, z.B. Mexiko, Brasilien.

Die Begründung für den »peripheren« Charakter des neuen Fordismus in der Dritten Welt lautet: "Wir glauben, daß es sich um einen authentischen Fordismus handelt, der auf einer Kombination von intensiver Akkumulation und Marktexpansion basiert. Aber er bleibt in dem Maße peripher, wie in den weltweiten Kreisläufen industrieller Branchen Arbeitsplätze und Produktionssegmente, die an qualifizierte Arbeitsprozesse - vor allem auf Ingenieurniveau - gebunden sind, im ganzen außerhalb dieser Länder verbleiben « (Lipietz 1984, 101). Lipietz zeichnet ein sehr differenziertes Bild der Peripherie, das zugleich exogene wie endogene Variablen berücksichtigt: »...die Dritte Welt ist heute eine Konstellation spezieller Fälle» (Lipietz 1984, 103).

Auffällig ist allerdings, daß Lipietz trotz seiner gelungenen Darstellung der Komplexität des Industrialisierungsprozesses in den Entwicklungsländern auf durchaus überflüssige Anleihen bei der Dependenztheorie nicht verzichtet. Wie in den Denkmodellen der Zentrum-Peripherie-Variante, sind die neuen Industrialisierungsprozesse in den Entwicklungsländern auch bei Lipietzletztlich fundamentalen Veränderungen im Zentrum geschuldet, nämlich der Krise des Fordismus, der aufgrund des Gesetzes vom tendenziellen Falls der Durchschnittsprofitrate nach neuen Verwertungsfeldern suche und sie in dem Schwellenländern finde. Eine Denkschablone, die im Widerspruch zu, den realen internationalen Kapitalströmen in den 70er Jahren steht und die den dependenztheoretischen Fehler wiederholt, die Peripherie als in letzter Instanz außeninduziert zu begreifen. Ferner kommt auch Lipietz nicht umhin, die neuen Akkumulationsprozesse in den Entwicklungsländern als "peripheral« zu klassifizieren. Als begrenzt, weil angeblich den Werkzeugmaschinenbau aussparend. Obwohl Lipietz für diese Schranke keine akkumulationstheoretische Begründung liefert, eine Schranke, die übrigens von den asiatischen Schwellenländern gerade mit äußerst hohen Wachstumsraten im Export von Werkzeugmaschinenbauprodukten mit hohem Technologiegehalt niedergerissen wird (IFO 1984), reproduziert er auch hier das bekannte Argumentationsmuster der Dependenztheorie. Reale ökonomische Fortschritte in den Entwicklungsländern werden von den alten Denkschemata vereinnahmt, indem man ihnen das Adjektiv "peripher « óder 
"neu « beifügt - "periphere « Akkumulation, "neuer « Fordismus oder "neue« internationale Arbeitsteilung, »neue« technologische Abhängigkeit - und dadurch suggeriert, im Grunde handle es sich nur um eine Variante altbekannter Grundmuster.

Doch wie bereits gesagt, abgesehen von diesen beiden hereditären Argumentationsmängeln, ist Lipietz Beitrag jenen Versuchen beizuordnen, die ausgehend von den Defiziten der Dependenztheorie sich um eine differenzierte Reformulierung der Unterentwicklungsproblematik bemühen.

\section{Schlußbemerkungen}

Wallersteins Versuch, auf der Basis des Begriffs »kapitalistische Weltwirtschaft « eine Weltsy. stemtheorie zu formulieren, die die weltgeschichtliche Entwicklung seit dem 16. Jahrhundert erklärt, ist aus folgenden Gründen als gescheitert zu betrachten:

- Er vermag die Systeme des Spärfeudalismus (Absolurismus), des Industriekapitalismus und des »Realsozialismus« nur um den Preis eines oberflächlichen Kapitalismusbegriffs, der von den Produktionsverhältnissen abstrahiert, als Subsysteme bzw. Epochen der kapitalistischen Weltwirtschaft zu interpretieren.

- Er analysiert die Struktur der kapitalistischen Weltökonomie mit logisch inkonsistenten und empirisch irrelevanten Begriffen des »ungleichen Tausches«.

- Er legt eine ökonomistische Interpretation der Hegemoniesequenzen im internationalen System seit dern 16. Jahrhundert vor, die einer empirischen und theoretischen Überprüfung nicht standhält.

Wallerstein reproduziert damit die Schwächen der internationalen Dependenzdiskussion seit Mitte der 60er Jahre. Versuchte diese, die Unterentwicklungsproblematik unter Zugrundelegung des Zentrum-Peripherie-Modells zu erklären, transponiert Wallerstein dieses Paradigma nicht nur auf die kapitalistische Weltwirtschaft in ihrer Totalität - also unter Einschluß der Beziehungen zwischen den kapitalistischen Industrieländern -, sondern auch auf historische Verhältnisse, die dem industriekapitalistischen Weltsystem vor- und nachgelagert sind - also das System des Spätfeudalismus und des »Realsozialismus«. Wallerstein übernimmt mit dem dependenztheoretischen Modell eine theoretische Konstruktion, die sich bereits in der Unterentwicklungsproblematik als unzureichend erwiesen hat, deren Tragfähigkeit aber vollends dadurch überfordert wird, daß ihr in der Weltsystemversion eine noch größere historische und weltwirtschaftliche Reichweite zugesprochen wird. Angesichts dieser eklatanten Mångel der Theorie Wallensteins stellt sich die Frage nach den Ursachen des regen Interesses, das die Weltsystemtheorie international erweckt hat. Eine Antwort ist m. E. in der begrifflichen Struktur der Weltsystemtheorie, eine andere im politischen Bedürfnis der Linken nach einer Theorie des internationalen Klassenkampfes zu finden.

Die begriffliche Struktur der Theorie Wallersteins ist durch folgende Eigenschaften gekennzeichnet:

a) Sie enthält sehr wenige Schlüsselbegriffe (Kapitalismus, Zentrum, Semiperipherie, Peripherie, ungleicher Tausch, ungleiche Arbeitssysteme, Hegemonie), die wiederum durch äußerst einfache Beziehungen verknüpft sind, letztlich durch den Nexus von Profit und Macht sowie von stark/qualifiziert versus schwach/unqualifiziert.

b) Diese Kategorien sind so allgemein definiert, daß sie ungemein viele historische und gesellschaftliche Daten umfassen, eine große Reichweite haben. 
Eine Theorie, die diese Eigenschaften aufweist, die also mit wenigen, einfachen und umfassenden Begriffen operiert, ist äußerst attraktiv, weil sie in einer chaotischen Welt voller Widersprüche durch Komplexitätsreduktion scheinbar Sicherheit und Gewißheit offenbart. Mit Wallerstein können wir vom 16. bis 20. Jahrhundert alle nationalen und internationalen Ereignisse politischer, ökonomischer, militärischer, religiöser, kultureller Art unter einen Begriff subsumieren: das Weltsystem. Mit einer solchen Zauberformel im Tornister können wir ruhigen Gewissens unwissend mit Meilenschritten durch die Weltgeschichte eilen, denn wir wissen heute wie gestern und morgen: es ist immer das Weltsystem.

Neben Sicherheit und Gewil heit bietet Wallerstein der internationalen Linken noch eine wejtere Faszination: eine internationalistische politische Perspektive. Das internationale System gliedert sich in starke, mittelstarke und schwache Staaten, die über einen Ausbeutungsnexus verknuipft sind. Diese Null-Summen-Optik erleichtert Parteilichkeit, Moral und Unmoral scheiden sich säuberlich. Weltmarkttheorien, die Ausgleichsmechanismen in internationalen Systemen behaupten, oder Unterentwicklungstheorien, die endogene Ursachen ins Zentrum der Analyse rücken, wirken gegenüber diesem einfachen politischen Grundmuster desorienticrend. Die Dependenztheorie führt gegen alle Empirie und gegen alle kritischen Kririkaster ein zähes Leben, weil sie in einer komplexen Welt das Bedürnnis nach Gewißheit und einer klaren politischen Perspektive befriedigt. Wer würde sich nicht wehren, wenn ihm beides genommen werden soll?

\section{Anmerkungen}

1 An diesen Ausführungen zum Verhältnis von Politik und Ökonomie ist kritisiert worden, die Separierungsthese übersehe die vielfältigen Interventionen des kapital istischen Staates in die Ökonomie, wobei zudem die Unterscheidung zwischen Binnen-und Weltmarkt nicht einsichtig sei. Die Kritik basiert auf zwei Mißverständnissen:

a) DieSeparierungsthese ist für die kapitalistische Produktionsweise in Abgrenzung zu vorkapitalistischen Produktionsweisen, in denen die Instanzen Politik und Ökonomie stets verschmolzen sind, formuliert. Das System freier Lohnarbeit ermöglicht in der kapicalistischen Produktionsweise diese strukturale Separierung. Z war sind auch unter kapitalistischen Bedingungen Staatsinterventionen in die Ökonomie systemnotwendig, diese sind aber von der staatlichen Präsenz in der Ökonomie vorkapitalistischer Produktionsweisen struktural zu unterscheiden. Ohne dieses zentrale Abgrenzungskriterium würden die Grenzen zwischen den verschiedenen Produktionsweisen verschwimmen.

b) Nationale und internationale Staatsinterventionen sind begrifflich auseinanderzuhalten, weil die Instanz Politik auf dem Weltmarkt im Unterschied zum nationalen Markt prinzipiell über mindestens Wechselkurse, aber auch Zölle, NTB's in die einzel wirtschaftlicbe Konkurrenz eingreift. Dieser Unterschied entspringt eben der Form des Staates als Nationalstaat.

2 Seit David Ricardo das Theorem der komparativen Kosten formuliert hat, ist sich die traditionelle Außenwirtschaftstheorie der Differenz zwischen Binnen- und Weltmarktkonkurrenz bewußt. Das Theorem der komparativen Kosten und das Theorem der modifizierten. Wirkungsweise des Wertgesetzes auf dem Weltmarkt implizieren beide den Schutzmechanisnuus der Wechselkurse. Ein großer Teil der neomarxistischen Weltmarkttheorie weigert sich, diese Zusammenhänge anzuerkennen, weil dabei einige Prämissen der Marxschen Geldtheorie geopfert werden müssen. Resultat sind dann Weltmarktanalysen, die immer noch ein metallistisches Weltwährungssystem unterstellen (z. B. die Sost 1981 oder Siegel 1980), und internationale Ausbeutungstheorien, die stets an der Beantwortung der Frage scheitern, weshalb sich die ausgebeuteten weniger produktiven Natio- 
nen ökonomisch rascher entfalten können als die ausbeutenden Hegemonialmächte. Für die Stellung der Entwicklungsländer in der Weltwirtschaft gilt das Modifikationstheorem allerdings nur mit Einschränkung (vgl. dazu Busch/Gruner/Tobergte 1984, 78 f.).

3 Zur Überproduktionstheorie Wallersteins, die stark an Rosa Luxemburgs Theorie der »dritten Person « erinnert, vgl. auch Wallerstein 1984, 6 ff., $16 \mathrm{ff}$., 53 f.

4 Einen weiteren Versuch, lange Wirtschaftszyklen mit langen Weltpolitikzyklen analytisch zu verbinden, hat G. Modelski unternommen (Modelski 1978 und 1981). In Anlehnung an Kondratieff/ Rostow unterscheidet Modelski lange Aufschwungs- und Abschwungsphasen eines Preisindexes. Hausse-Perioden des Preisindex (1792-1815, 1848-1873, 1914-1946, 1974-?) ordnet er Innovationen oder Umbrüche im internationalen politischen System zu, Baisse-Perioden (1763-1792, 1815-1848, 1873-1913, 1946-1973) Innovationen oder Umbrüche im ökonomischen Sektor (z.B. das Aufkommen neuer Wachstumsbranchen). Weltkriege ereignen sich in jeder zweiten Hausse-Periode (Napoleonische Kriege, die beiden Weltkriege). Dieses »altering innovation« model begründet Modelski wie folg:

Es könnte sein, daß die Weltpolitik und die Weltökonomie 'dem selben Trommler lauschen': beide sind durch das selbe Preis-Wert-Prioritätssystem koordiniert, beide reagieren auf die selben Veränderungen im sozialen Wertsystem. Wenn die Nachfrage nach politischen Gütern und Dienstleistungen (z. B. Waffen) und nach besonderen politischen Innovationen steigt, wird die Knappheit der Ressourcen ... wachsen und das generelle Preisniveau wahrscheinlich ebenfalls ansteigen. Wenndie Nachfrage nach Waffen zurückgeht, würden wir eine Reduktion der inflationären Zwänge erwarten. Aus diesen Gründen würden wir erwarten, daß sich die Nachfrage nach grundlegenden Ressourcen, also auch das generelle Preisniveau, im großen und ganzen in derselben Weise verändert wie die Nachfrage nach politischen Gütern und Dienstleistungen im Weltsystem « (Modelski 1981, 75 f.).

Auch dieses politisch-ökonomische Zyklus-Modell provoziert eine Reihe von Fragen. Am wichtigsten scheinen mir folgende zu sein:

1. In welcher Beziehung steht der Zyklus des Preissystems zu langen Konjunkturzyklen? Auffällig ist, daß Modelskis Baisse-Periode von 1873-1913 eine B-Phase(1873-1893) und eine A-Phase (1893-1913) langer Wachstumszyklen überdeckt; ebenso fällt in die Hausse-Periode von 1914 1946 eine B-Phase des langen Kondratieff-Zyklus (1913-1939).

2. Sind nicht die Wachstumszyklen für die Analyse von Strukturproblemen des ökonomischen Systems bedeutsamer als die Preiszyklen? Macht es z. B. Sinn, die Weltwirtschafrskriese im Jahre 1929 innerhalb einer Hausse-Periode des Preiszyklus unter den Tisch fallenzulassen?

3. Läß sich die These aufrechterhalten, daß in Hausse-Perioden keine wichtigen ökonomischen Innovationen statffinden? Wie sind z. B. die wichtigsten technologischen Basisinnovationen der $30 \mathrm{er}$ Jahre dieses Jahrhunderts zu beurteilen?

4. Läßt sich ernsthaft behaupten, Baisse-Perioden würden keine wichrigen politischen Innovationen im internationalen System kennen? Wie ist in diesem Zusammenhang z. B. der Entkolonialisierungsprozeß der 50 er und 60er Jahre, die Entspannungspolitik zwischen den beiden Supermächten seit Beginn der 60er Jahre und und die Gründung von EWG und EFTA Ende der 50er Jahre zu beurteilen?

Einer ernsthaften Prüfung dieser Frage hält das »altering innovation« model von G. Modelsky m. E. nicht stand. Es basiert auf einer problematischen ökonomischen Variablen (dem Preisindex), eskamotiert entscheidende ökonomische Ereignisse (Weltwirtschaftskrise 1929) und operiert mit nicht ausgewiesenen Selektionskriterien bei der Bestimmung politischer und ökonomischer Innovacionen. 


\section{Literatur}

AITHUSSER, Louis, 1968: Für Marx, Frankfurt/a.M.

AMIN, Samir, 1975: Die ungleiche Entwicklung, Hamburg

ANDERSON, Perry, 1978: Von der Antike zum Feudalismus - Spuren der Übergangsgesellschaften, Frankfurt/a.M.

DERS., 1979: Dic Entstehung des absolutistischen Staates, Frankfurt/a.M.

BARATI-NOVBARI, N. und SEELOW, F., 1982: Das Ende der Dritten Welt - Weltwirtschaftlicher Differenzierungsprozeß und Strukturwandel in den außereuropäischen Entwicklungsländern, Bochum

BLASCHKE, Jochen ( $\mathrm{Hg}_{\text {. }}$ ), 1983: Perspektiven des Weltsystems - Materialien zu Immanuel Wallerstein, »Das moderne Weltsystem«, Frankfurt/a.M.

BRENNER, Robert, 1983: Das Weltsystem - Theoretische und historische Perspektiven, in: Blaschke 1983

BUSCH, Klaus, 1973: Ungleicher Tausch - Zur Diskussion über internationale Durchschnittsprofitrate, ungleicher Tausch und komparative Kostentheorie anhand der Thesen von Arghiri Emmanuel, in: Prokla Nr. 8/9, West-Berlin

DERS., 1974: Die Multinationalen Konzerne - Zur Analyse der Weltmarktbewegung des Kapitals, Frankfurt/a.M.

DERS., 1978: Die Krise der Europäischen Gemeinschaft, Frankfurt/a.M.

BUSCH, K./GRUNERT, G./TOBERGTE, W.,1984: Strukturen der kapitalistischen Weltökonomie - Zur Diskussion der Weltmarktbewegung des Kapitals, Saarbrücken

CHATELAIN, E., 1974: Wohin führt die These vom ungleichen Tausch?, in: Kritik der politischen Ökonomie 2, West-Berlin

DEUBNER/REHFELDT/SCHLUPP/ZIEBURA (Hrsg.), 1979: Die Internationalisierung des Kapitals - Neue Theorien in der internationalen Diskussion, Frankfurt/a.M. / New York

EIKENBERG, Armin, 1983: Die paradignatische Krise der Theorie ökonomisch unterentwickelter Gesellschaftsformationen. Ein Versuch der Neuformulierung der Problematik des sog. „Peripherie-Kapitalismus«, Diss., vorgelegt am Fachbereich Sozialwissenschaften der Universität Osnabrück

EMMANUEL, A., 1972: L'échange inégal, Paris

FRANK, Andre Gunder, 1980: Abhängige Akkumulation und Unterentwicklung, Frankfurt/a.M.

HURTIENNE, Th., 1981: Peripherer Kapitalismus und autozentrierte Entwicklung - Zur Kritik des Erklärungsansatzes von Dieter Senghaas, in: Prokla Nr. 44, West-Berlin

IFO, 1984: Die asiatischen Schwellenländer - Eine Gefahr für die deutsche Investitionsgüterindustrie?, in: ifo-schnelldienst Nr. 28/84, München

KI.EINKNECHT, A., 1984: Innovationsschübe und lange Wellen: Was bringen »Neo-Schumpeterianische« Kriseninterpretationen?, in Prokla Nr. 57, West-Berlin

KRIEDTE, Peter, 1980: Spätfeudalismus und Handelskapital. Grundlinien der europäischen Wirtschaftsgeschichte vom 16. bis zum Ausgang des 18. Jahrhunderts, Göttingen

LAIR, J., 1974: Ungleicher Tausch und "Third-Worldism«, in: Kritik der politischen Ökonomie 2, West-Berlin

LIPIETZ, Alain, 1984: Imperialism or the beast of the apocalypse, in: Capital and Class Nr. 22/84

MANDEL, Ernest, 1983: Die langen Wellen im Kapitalismus, Frankfurt/a.M.

MODELSKY, G., 1978: The Long Cycle of Global Politics and the Nation-State, in: Comparative Stu * dies in Society and History, Nr. 20/78

DERS., 1981: Long Cycle, Kondratieffs, and Altering Innovations: Implications for US Foreign Policy, in: Kegley, Ch.W., und McCowan, P., 1981: The Political Economy of Foreign Policy Behavior, Beverly Hills

SCHWEERS, Rainer, 1980: Kapitalistische Entwicklung und Unterentwicklung, Frankfurt/a.M.

SENGHAAS, Dieter (Hrsg.), 1974: Peripherer Kapitalismus - Analysen über Abhängigkeit und Unterentwicklung, Frankfurt/a.M. 
DERS., 1977: Weltwirtschaftsordnung und Entwicklungspolitik - Plädoyer für Dissoziation, Frankfurt/a.M.

DERS., (Hrsg.), 1979: Kapitalistische Weltökonomie - Kontroversen über ihren Ursprung und ihre Entwicklungsdynamik, Frankfurt/a.M.

DERS., 1982: Von Europa lernen - Entwicklungsgeschichtliche Betrachtungen, Frankfurt/a.M.

SIEGEL, Tilla, 1980: Kapitalismus als Weltsystem - Methodische Probleme einer marxistischen Analyse des Weltmarkts, Frankfurt/a.M.

SKOCPOL, Theda, 1977: Wallerstein's World Capitalist System. A Theoretical and Historical Critique, in: American Journal of Sociology 1982/5, 1075-1090

SOZIALISTISCHE STUDIENGRUPPEN (Sost), 1981: Kapitalistische Weltwirtschaft, Hamburg

SWEEZY, Paul (Hrsg.), 1978: Der Übergang vom Feudalismus zum Kapitalismus, Frankfurt/a.M.

TOBERGTE, Walter, 1984: Die Internationalisierung des Kapitals in Form von Direktinvestitionen Ursachen und Konsequenzen für die Gewerkschaften, in: WSI-Mitteilungen 4/84, Düsseldorf

WALLERSTEIN, Immanuel, 1974: The Modern World System. Capitalist Agriculture and the Origins of the European World-Economy in the Sixteenth Century, London

DERS., 1979a: The Capitalist world-economy, Cambridge

DERS., 1979b: Aufstieg und künftiger Niedergang des kapitalistischen Weltsystems. Zur Grundlegung vergleichender Analyse, in: Senghaas, 1979

DERS., 1980: The Modern World-System II. Mercantilism and the Consolidation of the European World-Economy, 1600-1750, London

DERS., 1984: The Politics of the World-Economy, Cambridge University Press, London und New York

WARREN, Bill, 1973: Imperialism and Capitalist Industrialization, in: new left review Nr.81, London

WORSLEY, Peter, 1983: Drei Welten oder Eine? Eine Kritik der Weltsystemtheorie, in: Blaschke, 1983 